\title{
Recent developments in MEKC-MS
}

K. Otsuka and S. Terabe

Department of Material Science, Faculty of Science, Himeji Institute of Technology, Kamigori, Hyogo 678-1297, Japan

Recent developments in on-line coupling of micellar electrokinetic chromatography with mass spectrometry (MEKC-MS) are described. Three kinds of techniques have been introduced to achieve MEKC-MS: (1) partial-filling MEKC, (2) MEKC using a high-molecular-mass surfactant, and (3) atmospheric pressure chemical ionization interface.

\section{Introduction}

Capillary electrophoresis (CE) has become popular as an analytical separation method owing to its high resolution and microscale capabilities. In most CE separations carried out with commercially available automated instruments, UV including UV diode-array is used as a detection scheme.
These optical absorbance detection systems, however, are sometimes not sensitive nor specific for highly complex samples or minute sample quantities.

Recently, mass spectrometry (MS) has been recognized as one of the most powerful detection methods of CE due to the high sensitivity, high selectivity, and also the availability of structural information. Number of reports on on-line coupling of CE with MS (CE-MS) have been published. In most CE-MS systems, an electrospray ionization (ESI) is employed as an interface between a CE instrument and an MS detector. ESI is suitable for the on-line coupling of MS with liquid phase separation techniques such as HPLC and $\mathrm{CE}$, because gas-phase ions of the analyte are produced efficiently under the atmospheric pressure [1].

Micellar electrokinetic chromatography (MEKC) is one of the most popular techniques in CE and is capable of separating neutral compounds as well as charged solutes by using a separation solution containing an ionic surfactant micelle. Basically MEKC uses the same instrument as other 
CE techniques, so that the same problem applies to MEKC concerning the photometric detection and on-line coupling of MEKC with MS is one of attractive detection schemes in MEKC as well as in CE.

The development of MEKC-MS systems, however, have not successfully progressed in last couple of years, since most surfactants normally used in MEKC often deteriorate ionization efficiency and also cause high background signals in ESI-MS. That is, conventional MEKC system using an ionic low-molecular-mass surfactant (LMMS) micelle, e.g., sodium dodecyl sulfate (SDS), as a pseudo-stationary phase is hard to be coupled with ESI-MS: SDS generates stable sodium attached ions in the positive ionization mode in ESIMS.

To overcome these limitations on MEKC-MS several methods has been developed: (1) the use of the partial filling (PF) technique (PF-MEKC), (2) the use of a high-molecular-mass surfactant (HMMS) instead of a conventional LMMS as a pseudo-stationary phase in MEKC, and (3) the use of the atmospheric pressure chemical ionization (APCI) method instead of ESI.

In this paper, recent progress in MEKC-MS using these three techniques are briefly reviewed along with some basic considerations and applications.

\section{Partial-filling MEKC-MS (PF-MEKC-MS)}

The partial-filling (PF) technique was first developed to use a non-UV-transparent compound as an additive to the separation solution in $\mathrm{CE}$ [2]. Then the PF technique was applied to the enantiomer separations by capillary zone electrophoresis using proteins as chiral selectors [3], and the technique has been adapted for MEKC-ESI-MS [4-6]. Figure 1 shows the schematic illustration of PF-MEKC: The separation capillary is first filled with a buffer containing no micelle, followed by the introduction of a micellar zone. And then a sample solution containing two components is injected. By applying a high electric field to the capillary, the MEKC separation is performed while the sample passes through the micellar zone and the separated analytes mig rate into the buffer zone without the surfactant. Finally, the separated zone reaches the detection end, whereas the micellar

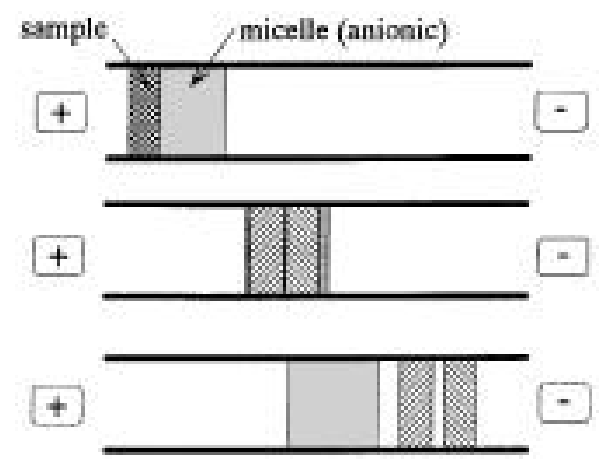

Figure 1. Schematic illustration of the partial-filling MEKC (PFMEKC) technique: The sample solution contains two components. (Reprinted from the Ph. D. Thesis of Ozaki, H., Kyoto University, March 1998). zone does not reach the detection area yet. That is, by stopping the high electric field at this time, the micellar zone does not reach the detector cell. As for the case in MEKCMS, we can suppress the introduction of the micellar zone into the ESI interface by cut-off of the electric field after the introduction of the sample zone into the interface.

The first paper of PF-MEKC-ESI-MS appeared in 1995 as a potential technique for MEKC-MS [4]. Recently, several papers on the capability and mechanism of PF-MEKCMS have been published [5-8,17]. For example, three closely related neurotensin analogs, [D-Tyr $\left.{ }^{11}\right]-,\left[\mathrm{D}-\mathrm{Phe}^{11}\right]-$, and [D$\operatorname{Trp}^{11}$-neurotensins, were successfully separated and detected by PF-MEKC-ESI-MS using sucrose monododecanoate as a pseudo-stationary phase [6], as shown in figure 2, obtained with a laboratory built ESI interface, a Jasco CE-990 CE instrument, and a modified Hitachi M1000 quadrupole MS detector. In CE-ESI-MS, a sheath
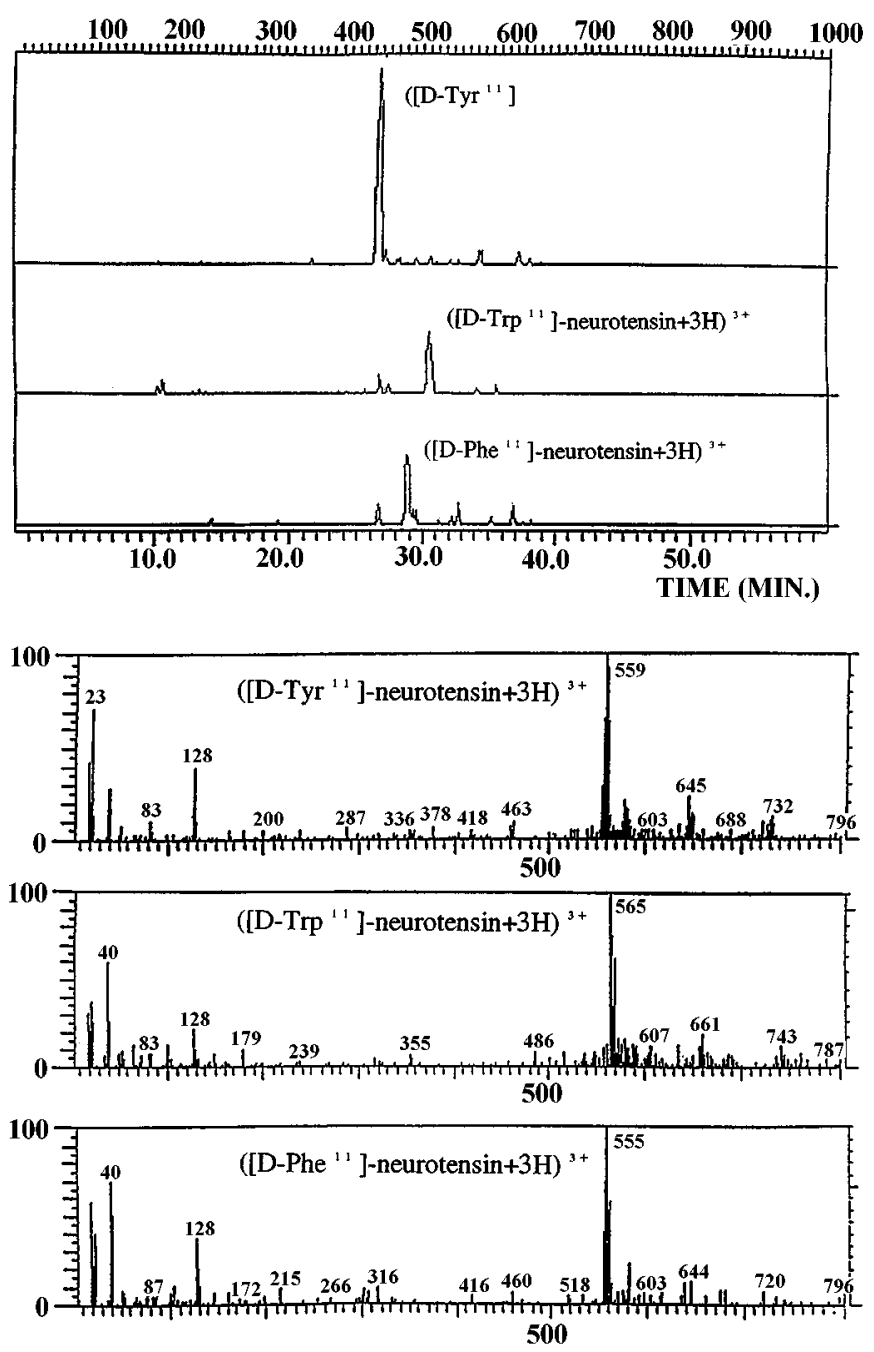

Figure 2. Single-ion electropherograms a) and mass spectra b) of three neurotensin derivatives by PF-CE-ESI-MS using sucrose monododecanoate. Running buffer, $30 \mathrm{mM}$ ammonium

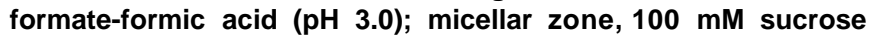
monododecanoate in the running buffer filled at 50 mbar for $15 \mathrm{~min}$; capillary, $50 \mu \mathrm{m}$ i.d. $\times 100 \mathrm{~cm}, 150 \mu \mathrm{m}$ o.d.; CE applied voltage, $17 \mathrm{kV}$; electrospray voltage, $3 \mathrm{kV}$; sheath liquid flow, water-methanol formic acid (50:50:1, v/v/v) at $1 \mu \mathrm{L} / \mathrm{min}$. (Reprinted from Ref. 6). 
liquid flow is normally required to make the electric contact with running solution and to make an electrospray stable. Here, the coaxial sheath flow was delivered by an HPLC pump at normally 1 to $10 \mu \mathrm{L} / \mathrm{min}$. Since the composition and flow rate of the sheath liquid sometimes affects the MS intensity and stability, one must optimize the composition of the sheath liquid flow. A mixture of water and methanol containing small amount of acid, e.g., formic acid or acetic acid, is usually used for the sheath liquid. In figure 2, a watermethanol-formic acid (50:50:1, v/v/v) was used as a sheath liquid. It should be noted that the example in figure 2 is not the MEKC separation exactly, since the surfactant used was neutral.

Although in the PF-MEKC the separation window is narrower than the normal MEKC system [8], sufficient separation was attained by optimizing the micellar zone length. As for another example, separation and detection of caffeine and ethenzamide was reported by using SDS-PF-MEKC-MS along with the theoretically treatment of PF-MEKC [8].

\section{HMMS-MEKC-MS}

There have been some papers on HMMS-MEKC [9-16], in which sodium 10-undecenyl sulfate (SUS) oligomer, sodium undecylenate oligomer (SUA), butyl acrylate-butyl methacrylate-methacrylic acid copolymer sodium salt (BBMA), and diisobutylene-maleic acid copolymer sodium salt (DMA) were used as pseudo-stationary phases. The critical micelle concentration (CMC) of HMMS should be effectively zero and hence, HMMS is expected to be an ideal pseudo-stationary phase of MEKC coupled with ESI-MS because of the stability or the formation of the micelle at low surfactant concentration. Also, in contrast to a conventional LMMS such as SDS, the HMMS micelle will hold unimolecular structure even in ESI-MS since it is single covalently bonded.

In a BBMA-MEKC-ESI-MS system, some quaternary ammonium salts, alkaloids, and sulfamides [14] and three xanthine derivatives [15] (Fig. 3) were successfully sepa-
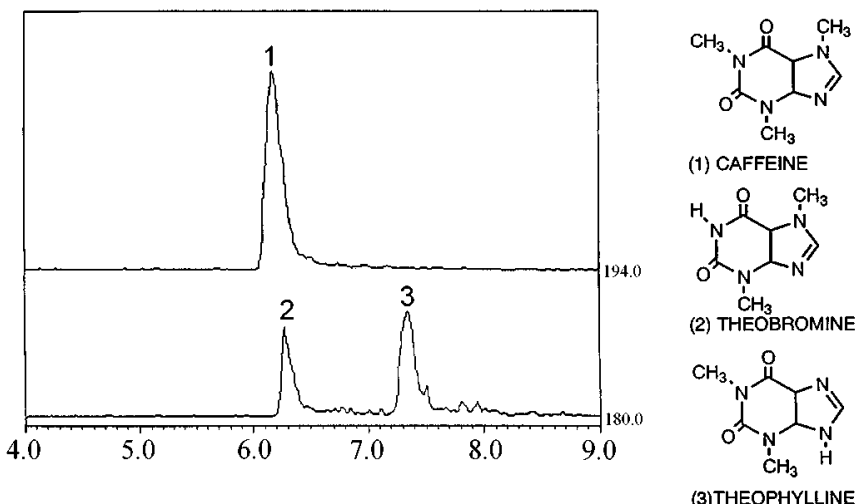

Figure 3. An HMMS-MEKC-ESI-MS detection of three xanthine derivatives. Separation solution, $1 \%$ BBMA in $10 \mathrm{mM}$ ammonium formate buffer (pH 7) containing 10\% methanol; capillary, $50 \mu \mathrm{m}$ i.d. $\times 50 \mathrm{~cm}$; CE applied voltage, $13 \mathrm{kV}$; electrospray voltage, $3 \mathrm{kV}$; sheath liquid flow, water-methanol-formic acid (50:50:1, v/v/v). (From Ref. 15.) rated and detected. In DMA-MEKC-ESI-MS, a test mixture including phenyltrimethylammonium chloride, 1-naphthylamine, quinine sulfate, tetraphenylphosphonium chloride, and octaoxyethylenedodecanol was successfully separated and detected [16]. However, a decrease in the MS signal intensities were observed with an increase in the concentrations of both BBMA and DMA [16,17]. Details of the ionization mechanism of ESI are complex and some theoretical studies have been reported [18], although the effects of surfactants on the ESI efficiency and mechanism along with the other factors of the separation solution, i.e., the salt concentration, $\mathrm{pH}$, and the organic solvent content, are not clarified yet. The difference in ESI mechanisms between HMMS and LMMS was also experimentally proposed [19].

\section{MEKC-APCI-MS}

The use of the atmospheric pressure chemical ionization (APCI) method in CE-MS instead of ESI has been developed [20,21]. The APCI interface was originally designed for liquid chromatography-MS (LC-MS). The APCI interface between $\mathrm{CE}$ and MS consists of an electrospray-type nebulizer, a vaporizer, and an APCI source using needle electrode for the corona discharge. Note that the APCI system is called electrospray-chemical ionization (ES-CI) interface by the inventors [20]. In the APCI mode, molecules are ionized by the corona discharge followed by ion-molecule reactions. When a make-up solution or sheath liquid, e.g., methanol, is used, methanol is first ionized by the corona discharge and then the analyte molecule having a stronger proton affinity than methanol will be easily protonated by the ion-molecule reactions. The APCI interface should be useful for CE-MS including MEKC-MS because ionization processes in APCI are not significantly affected by the presence of salt in a background CE buffer. Takada et al. reported CE-APCI-MS using sodium phosphate buffer [20] and MEKC-APCI-MS using SDS [21]. This implies that conventional buffer systems, such as phosphate, borate, or tris, can be used in CE-APCI-MS.

In MEKC-APCI-MS, a micellar solution even containing SDS can be introduced directly into the ionization area of the MS detector without a severe decrease in sensitivity. First we successfully demonstrated the MEKC-APCI-MS separation and detection using BBMA as a pseudo-stationary phase [15]. The use of conventional LMMSs including SDS and sodium cholates as pseudo-stationary phases were also investigated in MEKC-APCI-MS [16]. Figure 4 shows an example of MEKC-APCI-MS of three xanthine derivatives, as the same as in figure 3, with SDS [15]. The successful separation and detection was obtained even in a $50 \mathrm{mM}$ SDS solution. Here, a phosphate buffer was used, and this also shows a usefulness of APCI.

Effects of some experimental conditions, such as micellar concentration, $\mathrm{pH}$, sheath liquid flow rate, desolvation temperature, and drift voltage, on APCI efficiency were investigated for both positive ionization-detection and negative ionization-detection modes in APCI. The drift voltage is applied after the desolvation process to produce a single ionized molecule by a collision. As a result, the SDS concentration and sheath liquid flow rate did not affect the MS intensities both in positive and negative modes. 


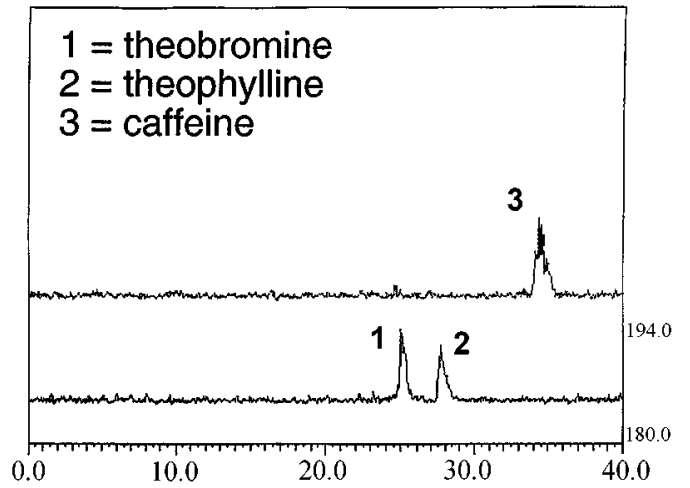

Figure 4. An SDS-MEKC-APCI-MS detection of three xanthine derivatives. Separation solution, $50 \mathrm{mM}$ SDS in $25 \mathrm{mM}$ phosphate buffer (pH 6.6); capillary, $50 \mu \mathrm{m}$ i.d. $\times \mathbf{4 0} \mathrm{cm}$; applied voltage, $5 \mathrm{kV}$; electrospray voltage, $3 \mathrm{kV}$; sheath liquid flow, methanol. (From Ref. 15.)

The signal-to noise ratio was not affected by the change in the SDS concentration. The desolvation temperature and drift voltage, however, were found to be critical to the MS intensities, and optimal conditions were determined [22].

\section{Conclusion}

MEKC-MS is a promising technique especially for separating neutral analytes by $\mathrm{CE}$ and obtaining the highly sensitive and specific detectability. By using the PF-MEKC and HMMS-MEKC techniques, MEKC-ESI-MS can be established. APCI is a useful technique coupling MEKC with MS even when an ionic LMMS surfactant is used as a pseudostationary phase, as well as CE-MS without micelles. Of course, these three techniques can be combined, for example, as PF-MEKC-APCI-MS [23].

Since our work presented was performed rather old mass spectrometer and the sensitivity was lower than we expected. However, modern mass spectrometers can give much higher sensitivity at least an order of magnitude and rapid progresses on instrumentation will bring much higher sensitivity in near future.

\section{Acknowledgments}

The authors are grateful to Drs. N. Matsubara and P.G. Muijselaar and Messrs. K. Koezuka and M. Yokoi of Himeji
Institute of Technology, to Mr. H. Ozaki of Kaneka Techno Research Co. Ltd., to Mr. Y. Tanaka of Nippon Boehringer Ingelheim Co. Ltd. for their helpful discussions and assistance. Parts of this work has been supported by the Grantin-Aids for scientific research (Nos. 07554040, 08640778, and 09304071) from the Ministry of Education, Science, Sports and Culture, Japan.

\section{References}

1. Smith, R. D.; Barinaga, C. J.; Udseth, H. R. Anal. Chem. 1988, 60, 1948-1952.

2. Valtcheva, L.; Mohammad, J.; Petterson, G.; Hjertén, S. J. Chromatogr. 1993, 638, 263-267.

3. Tanaka, Y.; Terabe, S. J. Chromatogr. A 1995, 694, 277-284.

4. Terabe, S.; Ozaki, H.; Takada, Y.; Sakairi, M.; Koizumi, H. Abstracts of Papers, Seventh International Symposium on High Performance Capillary Electrophoresis, Würzburg, Germany; 1995 ; p. 53.

5. Nelson, W. M.; Tang, Q.; Harrata, A. K.; Lee, C. S. J. Chromatogr. A 1996, 749, 219-226.

6. Koezuka, K.; Ozaki, H.; Matsubara, N.; Terabe, S. J. Chromatogr. B 1997, 689, 3-11.

7. Yang, L.; Harrata, A.; Lee, C. Anal. Chem. 1997, 69, 18201826.

8. Muijselaar, P. G.; Otsuka, K.; Terabe, S. J. Chromatogr. A 1998, 802, 3-15.

9. Palmer; C. P.; Khaled, M. Y.; McNair, H. M. J. High Resolut. Chromatogr. 1992, 15, 756-762.

10. Palmer, C. P.; Terabe, S. J. Microcol. Sep. 1996, 8, 115-121.

11. Palmer, C. P.; Terabe, S. Anal Chem. 1997, 69, 1852-1860.

12. Ozaki, H.; Ichihara,A.; Terabe, S. J. Chromatogr. A 1994, 680, 117-123.

13. Ozaki, H.; Ichihara,A.; Terabe, S. J. Chromatogr. A 1995, 709, 3-10.

14. Ozaki, H.; Itou, N.; Terabe, S.; Takada, Y.; Sakairi, M.; Koizumi, H. J. Chromatogr. A 1995, 716, 69-79.

15. Ozaki, H.; Itou, N.; Terabe, S.; Takada, Y.; Sakairi, M.; Koizumi, H. Presented at the 56th Bunseki Kagaku Toronkai, Osaka, Japan, May 1995; Paper 1D01.

16. Ozaki, H.; Terabe, S. Bunseki Kagaku 1997, 46, 421-427.

17. Ozaki, S.; Terabe, S. J. Chromatogr. A 1998, 794, 317-325.

18. Kebarle, P.; Tang, L. Anal. Chem. 1993, 65, 972A-986A.

19. Rundlett, K. L.; Armstrong, D.W. Anal. Chem. 1996, 68, 34933497.

20. Takada, Y.; Sakairi, M.; Koizumi, H. Rapid Commun. Mass Spectrom. 1995, 9, 488-490.

21. Takada, Y.; Sakairi, M.; Koizumi, H. Anal. Chem. 1995, 67, 1474-1476.

22. Yokoi, M.; Ozaki, H.; Otsuka, K.; Terabe, S. Abstracts of Papers, Seventeenth Symposium on Capillary Electrophoresis, Hachioji, Tokyo, Japan; The Division of Electrophoresis, The Japan Society for Analytical Chemistry: Tokyo, Japan, 1997; pp 35-36.

23. Muijselaar, P. G.; Otsuka, K.; Terabe, S. unpublished data, 1997. 